\title{
Avaliação da degradação de propriedades de forma de agregados
}

\author{
Evaluation of the degradation of aggregates \\ shape properties
}

\author{
Henrique Cavalcanti ${ }^{1}$, Iuri Bessa ${ }^{2}$, \\ Daniele Ibiapina ${ }^{1}$, Verônica Castelo Branco ${ }^{1}$
}

\author{
${ }^{1}$ Departamento de Engenharia de Transportes, Universidade Federal do Ceará - Campus do Pici, Bloco 703, Fortaleza, \\ CE, Brasil. \\ ${ }^{2}$ Departamento de Engenharia de Transportes, Escola Politécnica da Universidade de São Paulo - Av. Prof. Almeida \\ Prado, Travessa 2, No. 83, Cidade Universitária, São Paulo, SP, Brasil. \\ e-mail: henriqueppcavalcanti@ hotmail.com,daniele@det.ufc.br, veronica@det.ufc.br, iuribessa@usp.br
}

\section{RESUMO}

As propriedades de forma de agregados (forma, angularidade e textura superficial) influenciam a textura do revestimento asfáltico, parâmetro que está diretamente ligado à aderência pneu-pavimento, à estabilidade e à segurança rodoviária. Diante da importância de se estudar essas características, este trabalho tem como objetivo principal avaliar o efeito do desgaste de agregados nas suas propriedades de forma. Para tanto, as propriedades de forma de agregados de três diferentes tamanhos foram analisadas por meio do uso dos métodos tradicionais e através de uma técnica de Processamento Digital de Imagens (PDI). Essas propriedades foram analisadas com o uso do Aggregate Image Measurement System 2 (AIMS2), antes e após a alteração das mesmas a partir do uso do Micro Deval (MD) e do equipamento de abrasão Los Angeles (LA), além do ensaio convencional de obtenção de Volume de Vazios (VV) não compactados. A angularidade das partículas diminuiu durante o processo de degradação, independente do equipamento utilizado nas análises. A esfericidade dos agregados desgastados no MD não sofreu variações significativas. Esse fato pode ter acontecido devido à pequena carga abrasiva utilizada no ensaio. A textura superficial, avaliada após o ensaio de abrasão Los Angeles, não apresentou variação significativa, uma vez que nesse ensaio há uma tendência de quebra das partículas dos agregados, fato que está mais relacionado à sua forma. Além disso, os resultados encontrados mostraram que o tamanho dos agregados e a presença de água influenciaram o processo de desgaste dos agregados. A combinação da caracterização por imagem das propriedades de forma de agregados e a utilização de processos mecânicos laboratoriais que sejam capazes de alterar essas propriedades pode se tornar uma solução adequada para a previsão do desempenho de pavimentos em relação à perda de aderência ao longo do tempo.

Palavras-chave: pavimentação asfáltica; agregados; propriedades de forma; degradação.

\section{ABSTRACT}

The aggregates shape properties (form, angularity, and surface texture) have an influence on the asphalt pavement surface texture, which is directly related to tire-pavement adhesion, to stability and to road safety. Due to the importance of studying these characteristics, this research has the main objective of evaluating the effect of aggregate degradation on their shape properties. In order to achieve this, the shape properties of aggregates with three different sizes were analyzed by means of traditional methods and also with the use of a Digital Image Processing (DIP) technique. These properties were analyzed with the use of the Aggregate Image Measurement System 2 (AIMS2), before and after the use of the Micro Deval (MD) and the Los Angeles abrasion equipment, besides the conventional test of uncompacted void content. The aggregates particles angularity values decreased during the degradation process for both equipment used in the analyses. The aggregates sphericity after the use of MD did not change significantly. This could be explained because smaller abrasive charges are used in this particular test. The surface texture evaluated after the Los Angeles abrasion test did not vary significantly, which can be explained due to the tendency of breakage during the test, a process that is more related changes in form conditions. Furthermore, the results showed that the ag- 
gregate size and the presence of water influenced the degradation processes of the aggregates. The combination of the characterization of aggregate shape properties by means of image techniques with the use of laboratorial mechanical processes that are capable of modifying these properties might be an adequate solution for the prediction of asphalt pavements performance in relation to adhesion loss throughout the time.

Keywords: asphalt pavements; aggregates; shape properties; degradation.

\section{INTRODUÇÃO}

Os agregados desempenham função importante na estrutura de um pavimento asfáltico, subcamadas e revestimento. Além dessa função estrutural, também influenciam a textura superficial do revestimento asfáltico, propriedade que está diretamente ligada à aderência pneu-pavimento, e que contribui para a segurança dos veículos ao rolamento. A passagem dos veículos ao longo da vida útil do pavimento asfáltico causa degradação (abrasão e quebra) e polimento dos materiais em campo.

A abrasão pode ser definida como perda de angularidade dos agregados, e a quebra está relacionada com fratura e perda de massa [1]. A verificação da perda das propriedades de angularidade e textura superficial de agregados, desgastados em equipamentos como o de abrasão Los Angeles (LA) ou o Micro Deval (MD), tem sido realizada como indicador da resistência dos agregados à abrasão e ao polimento, respectivamente. Em geral, quando há perda da textura superficial e de angularidade, após os testes realizados, há ainda perda da resistência à deformação permanente das misturas compostas pelos mesmos [2,3]

A aderência pneu-pavimento é influenciada por alguns parâmetros da mistura asfáltica, dentre os quais: a granulometria, as propriedades de forma e a resistência ao polimento dos agregados; o grau e o método de compactação utilizado na produção da mistura asfáltica. Esses parâmetros são determinantes para prover o atrito e a textura superficial do pavimento asfáltico [4]. Em relação à influência da textura superficial das partículas de agregados, MASAD et al. [5] e SHBEEB et al. [6] concluíram que agregados polidos tendem a gerar uma superfície mais lisa para as misturas asfálticas, consequentemente reduzindo as características de atrito da rodovia. HOU et al. [7] estudaram a aderência de misturas asfálticas com diferentes granulometrias e concluíram que uma distribuição descontínua dos agregados gera um coeficiente de atrito mais elevado em comparação com a distribuição bem-graduada. No que diz respeito à influência da compactação, GEORGIOU e LOIZOS [8] obtiveram o atrito de amostras de laboratório produzidas por diferentes métodos e concluíram que a compactação vibratória seguida da compactação estática melhora a textura das misturas asfálticas, em comparação com a compactação apenas estática ou apenas vibratória, porém esses resultados não necessariamente são observados em campo.

A textura superficial é um fator que desempenha forte influência nas propriedades de aderência do pavimento. De acordo com a ASTM E 867 (2012), há dois tipos de textura: a macrotextura, correspondente a dimensões cujo comprimento de onda é maior que $0,5 \mathrm{~mm}$ e a microtextura, correspondente a dimensões cujo comprimento de onda é menor que 0,5mm. Segundo ARAUJO [4], a microtextura tem suas propriedades ligadas à rugosidade da superfície do agregado, que pode ser classificada como lisa ou rugosa, e está diretamente ligada ao atrito. A macrotextura tem suas propriedades ligadas à distribuição das frações granulométricas dos agregados constituintes da mistura asfáltica e à relação com a velocidade de drenagem da água na superfície do pavimento.

No geral, são realizados testes de aderência em pista, após a construção das rodovias, para a verificação da conformidade ou não dos parâmetros de textura preconizados em normas e recomendações dos órgãos rodoviários. De acordo com MASAD et al. [9], o pêndulo britânico (ASTM E 303-93, 2013) é um dos métodos mais utilizados na medição da resistência à derrapagem, porém apresenta uma forma indireta de medida da textura dos agregados, em que as propriedades de atrito da amostra são os parâmetros considerados para avaliar a microtextura do material. Segundo os autores, esse teste desconsidera características que interferem nos seus resultados, como a distribuição granulométrica e o tamanho das partículas do agregado, além de que as manutenções periódicas no equipamento podem alterar seus resultados. Para a macrotextura, utiliza-se normalmente o ensaio de mancha de areia (ASTM E 965, 2015), no qual é obtido o parâmetro conhecido como altura da mancha de areia. Quanto maior o valor da altura, mais aberta ou mais grosseira é a macrotextura do pavimento.

O agregado sofre desgaste quando solicitado, e isso pode acarretar mudanças nas suas propriedades de forma [10]. BESSA et al. [3] avaliaram o comportamento do agregado após o desgaste no equipamento de abrasão Los Angeles. Os autores apresentaram uma metodologia dividida em três etapas: análise inicial dos agregados naturais através do uso do Processamento Digital de Imagens (PDI); desgaste dos agregados no equipamento de abrasão Los Angeles utilizando a norma brasileira; e, por fim, nova análise dos agregados 
após o desgaste. O objetivo era analisar como diferentes fontes de agregados se comportavam em relação à perda de angularidade e de textura superficial após o processo de desgaste no LA.

ARAUJO [4] avaliou o efeito da mudança nas propriedades de forma, dos agregados, no desempenho de misturas asfálticas e concluiu que o Aggregate Image Measurement System 2 (AIMS2) não tem sensibilidade para verificar alterações na macrotextura de misturas asfálticas quando as variações nos valores de angularidade são muito pequenas (menos que 10\%). O autor observou o mesmo comportamento se repete para a textura, porém quando a mudança ocorreu na distribuição granulométrica dos agregados, o AIMS2 conseguiu capturar variações dos valores de macrotextura. O autor constatou que, após o ensaio de abrasão Los Angeles, os agregados graúdos apresentavam maiores valores de esfericidade e menores valores de textura superficial, atribuindo os resultados obtidos aos processos de quebra, polimento e abrasão da superfície das partículas de agregados no equipamento utilizado. WANG et al. [11] investigaram o efeito da mudança na angularidade de agregados na resistência à deformação permanente. Foram testadas partículas de agregados antes da degradação no equipamento de Abrasão Los Angeles e após 200 a 1.200 ciclos de degradação (com incremento de 200). Foi obtida uma boa correlação do parâmetro de resistência com o parâmetro de angularidade, resultando em misturas mais resistentes aquelas que possuíam agregados menos degradados, ou seja, mais angulares, em sua composição.

No Brasil, não é comum a inclusão de análises do atrito e da textura e não há meios que permitam prever, em laboratório, essas propriedades, durante a fase de projeto de misturas asfálticas. Portanto, o projetista pode selecionar os agregados constituintes da mistura asfáltica a ser produzida, visando uma melhor aderência, a partir da granulometria e das propriedades de forma dos mesmos. Nos Estados Unidos, uma pesquisa realizada por GREER e HEITZMAN [12], em parceria Centro Nacional de Tecnologia de Asfalto, teve como principal objetivo propor um protocolo de laboratório para prever o desempenho de um pavimento asfáltico em relação à aderência e à derrapagem, por meio do uso combinado dos equipamentos MD e AIMS2. Entre as principais conclusões desse estudo, pode-se destacar que não há necessariamente uma boa correlação entre o desempenho de campo e a perda de textura em laboratório por meio do MD. Essa correlação pode depender principalmente do tipo de mineralogia do agregado testado.

A utilização do PDI se estabeleceu como ferramenta para análise das propriedades de forma de agregados e da estrutura interna de misturas asfálticas. O AIMS2 é um sistema de PDI, com mecanismo de aquisição de imagens e software, desenvolvido para analisar as propriedades de forma de agregados e as propriedades de superfície de misturas asfálticas compostas por eles. O sistema possui uma metodologia própria para classificação desses materiais [13]. A partir disso, este trabalho teve como objetivo principal avaliar o impacto das variações nas propriedades de forma de agregados com diferentes mineralogias, analisados após diferentes métodos de desgaste.

\section{MATERIAIS E MÉTODOS}

Para a realização do estudo, foram utilizados três agregados de Tamanhos Máximos Nominais (TMN) distintos (1", 3/4" e 1/2"), de mineralogia granítica, provenientes de uma pedreira localizada na Região Metropolitana de Fortaleza (RMF), no município de Caucaia (CE). Os agregados foram caracterizados quanto à granulometria e quanto às propriedades de forma, por métodos tradicionais e por meio do PDI. Os resultados de densidade foram: 2,62, 2,63 e 2,60 para os TMNs de 1", 3/4" e 1/2", respectivamente; para índice de forma, os valores obtidos foram $0,87,0,89$ e 0,86 para os materiais citados. A curva granulométrica dos agregados é mostrada na Figura 1. 


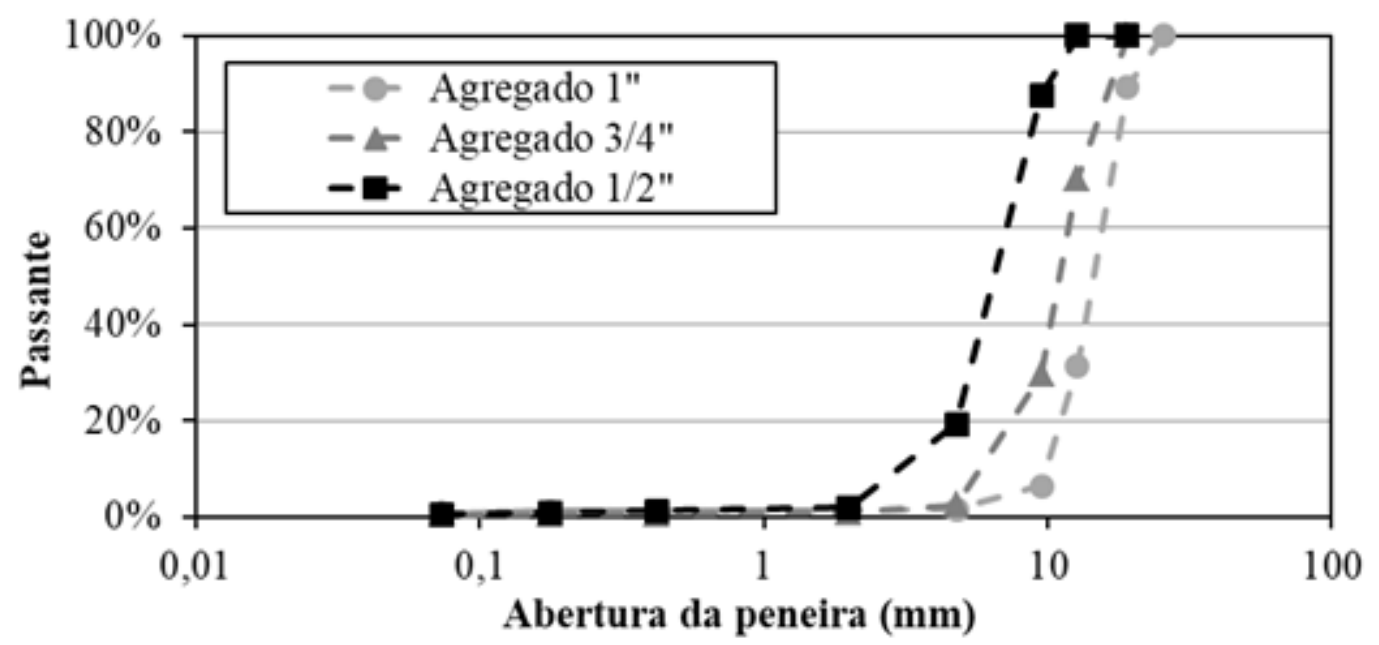

Figura 1: Distribuição granulométrica dos agregados utilizados.

A caracterização das propriedades de forma foi realizada com o uso do AIMS2, de forma separada para cada TMN. O AIMS2 é um equipamento desenvolvido para a obtenção das propriedades de forma, angularidade e textura de agregados graúdos e miúdos para uso em pavimentação asfáltica. $\mathrm{O}$ funcionamento do AIMS2 é dividido na análise de partículas graúdas (passantes na peneira de $25 \mathrm{~mm}$ e retidas na peneira de abertura de $4,75 \mathrm{~mm}$ ) e miúdas (passantes na peneira de $4,75 \mathrm{~mm}$ e retidas na peneira de $0,075 \mathrm{~mm}$ ). As partículas graúdas, no tal de aproximadamente 150, são posicionadas em uma bandeja transparente, separadas por tamanho de fração. Em seguida, a bandeja gira dentro do equipamento, e então é capturada a imagem de cada partícula da amostra. Na sequência, a bandeja gira novamente para medição da altura de cada partícula, totalizando a obtenção das três dimensões necessárias para as propriedades de forma e angularidade. Finalmente, uma última digitalização é realizada para obtenção dos níveis de textura de cada partícula, por meio do método das wavelets. Para as partículas miúdas, o material é espalhado uniformemente em uma badeja opaca, totalizando aproximadamente 150 partículas por fração de agregado. O equipamento faz a análise das partículas que não estejam em contato com nenhuma outra, resultando nos parâmetros desejados para a análise.

Além disso, foi adotada também a metodologia tradicional de análise dos parâmetros de forma e de textura dos agregados por meio do cálculo do volume de vazios de uma amostra não compactada. CROUCH e DUNN [14] utilizaram o método supracitado para validar duas metodologias de polimento de agregados. De acordo com esses autores, após o polimento do material, o volume de vazios da amostra não compactada tende a diminuir, uma vez que para o agregado polido é mais fácil encontrar uma disposição mais compactada.

O ensaio de abrasão Los Angeles (LA) para agregados destinados à pavimentação é normatizado pela norma DNER-ME 035 (1998). O Micro Deval (MD) foi desenvolvido na França na década de 1960 para avaliar a resistência à abrasão de agregados graúdos e miúdos. Ambos os equipamentos são comumente utilizados com o objetivo de se obter resultados baseados na perda de massa das amostras. O MD permite o uso de água durante o ensaio, para simular piores condições às quais os agregados são submetidos no pavimento. Não há normatização para a utilização desse equipamento no Brasil. A metodologia de ensaio de cada um desses dois equipamentos se difere principalmente em relação às esferas utilizadas e ao tempo de ensaio, apesar de ambos terem, no geral, a mesma carga abrasiva total $(5 \mathrm{~kg})$. No caso do LA, as esferas de aço utilizadas são maiores (aproximadamente 50mm de diâmetro em comparação com 10mm das esferas usadas no LA), e o tempo de rotação é reduzido (de 15 a 30min, em comparação com $2 \mathrm{~h}$ ), e velocidade de rotações do tambor também é menor (30\% da velocidade utilizada no MD). Essa redução no tamanho e pesos individuais das esferas faz com que o ensaio no MD seja menos severo no que diz respeito à quebra das partículas. A Tabela 1 apresenta as principais diferenças entre os dois ensaios de degradação mencionados. Devido às diferenças existentes entre os dois tipos de ensaios, não é possível correlacionar o nível de degradação gerados pelos dois equipamentos. Enquanto o MD tende a polir as partículas de agregados, o LA tende a quebrar suas arestas, ou seja, há uma tendência de perda de angularidade e maior perda de massa após o ensaio de LA, enquanto o ensaio realizado no MD tende a reduzir a textura superficial das partículas, com um menor nível de quebra. Além disso, agregados de fontes mineralógicas distintas podem ter comportamentos distintos para cada tipo de ensaio realizado. 
Tabela 1: Principais diferenças entre o ensaio realizado no Micro-Deval e no Abrasão Los Angeles.

\begin{tabular}{l|l|l}
\hline PROCEDIMENTO & MICRO-DEVAL & ABRASÃO LOS ANGELES \\
\hline Número de esferas & 12 & 11 \\
\hline Massa total das esferas $(\mathrm{g})$ & $5.000 \pm 5$ & $4.584 \pm 25$ \\
\hline Diâmetro das esferas $(\mathrm{mm})$ & 9 & 47,6 \\
\hline Velocidade do tambor (rpm) & $100 \pm 5$ & $32 \pm 1$ \\
\hline Diâmetro do tambor (mm) & 198 & 711 \\
\hline Comprimento do tambor (mm) & 174 & 508 \\
\hline Tempo total de ensaio (min) & 180 & 15 \\
\hline
\end{tabular}

$\mathrm{Na}$ presente pesquisa, o processo de desgaste dos agregados foi executado pelos dois equipamentos mencionados. Nas duas metodologias, foram obtidos os dados de perda de massa, em porcentagem, de cada fração de agregado analisada. Além disso, as variações nos valores das propriedades de forma de agregados analisados no AIMS e o volume de vazios não compactados da amostra. Na metodologia utilizada para o MD, os agregados foram desgastados em dois ambientes: seco e úmido (1 hora imerso em água antes do desgaste), e cada amostra foi avaliada ao longo de diferentes intervalos de tempo de polimento, similar ao que foi realizado por CROUCH e DUNN [14] .

Os resultados gerados através do uso do AIMS2, antes e depois dos processos de desgaste, foram analisados com base na distribuição das propriedades de forma, além de parâmetros estatísticos, e da classificação dos agregados. Maiores detalhes sobre a metodologia do trabalho podem ser encontrados em CAVALCANTI [15]. O AIMS2 possui duas propostas para sua normatização em andamento nos Estados Unidos, que foram submetidas à aprovação da American Association of State Highway and Transportation Officials, AASHTO (AASHTO TP81, 2010 e AASHTO PP64, 2010) e uma proposta de norma em desenvolvimento no Brasil, em fase de consulta pelo Instituto de Pesquisas Rodoviárias (IPR).

\section{RESULTADOS E DISCUSSÕES}

\subsection{Propriedades iniciais de forma}

A Tabela 2 apresenta os valores iniciais das propriedades de forma dos agregados analisados por meio do AIMS2, e a Tabela 3 traz a classificação dos parâmetros analisados a partir de duas metodologias: a classificação tradicional proposta por AL-ROUSAN [13] e a nova classificação proposta por IBIAPINA [16] para agregados brasileiros.

Tabela 2: Propriedades de forma obtidas por meio do AIMS2.

\begin{tabular}{l|l|l|l}
\hline TMN & ESFERICIDADE & TEXTURA SUPERFICIAL & ANGULARIDADE \\
\hline $1 "$ & 0,70 & 287 & 2.769 \\
\hline $3 / 4^{\prime \prime}$ & 0,67 & 197 & 2.884 \\
\hline $1 / 2^{\prime \prime}$ & 0,69 & 215 & 3.279 \\
\hline
\end{tabular}

Tabela 3: Classificação dos agregados a partir dos parâmetros de forma.

\begin{tabular}{l|l|l|l|l|l|l}
\hline \multirow{2}{*}{ TMN } & \multicolumn{2}{l|}{ ESFERICIDADE } & \multicolumn{2}{l|}{ TEXTURA SUPERFICIAL } & \multicolumn{2}{l}{ ANGULARIDADE } \\
\cline { 2 - 6 } & $\begin{array}{l}\text { Al Rousan } \\
(2004)\end{array}$ & $\begin{array}{l}\text { Ibiapina } \\
(2018)\end{array}$ & $\begin{array}{l}\text { Al Rousan } \\
(2004)\end{array}$ & $\begin{array}{l}\text { Ibiapina } \\
(2018)\end{array}$ & $\begin{array}{l}\text { Al Rousan } \\
(2004)\end{array}$ & $\begin{array}{l}\text { Ibiapina } \\
(2018)\end{array}$ \\
\hline $1 "$ & \multicolumn{2}{|l|}{ Baixa esfericidade } & Baixa rugosidade & Macio \\
\hline $3 / 4 "$ & Baixa esfericidade & Macio & Polido \\
\hline $1 / 2 "$ & Baixa esfericidade & Macio & Polido \\
\hline
\end{tabular}




\subsection{Análise das amostras desgastadas no Micro-Deval (MD)}

\subsubsection{Perda de massa e volume de vazios não compactados}

O Volume de Vazios (VV) não compactados é um parâmetro utilizado para estimar indiretamente as propriedades de forma, textura e angularidade. nesta pesquisa, esse parâmetro foi obtido e comparado com os resultados do AIMS2. A Figura 2 apresenta o comportamento desse parâmetro ao longo do processo de polimento das amostras para os três tamanhos de agregados analisados.

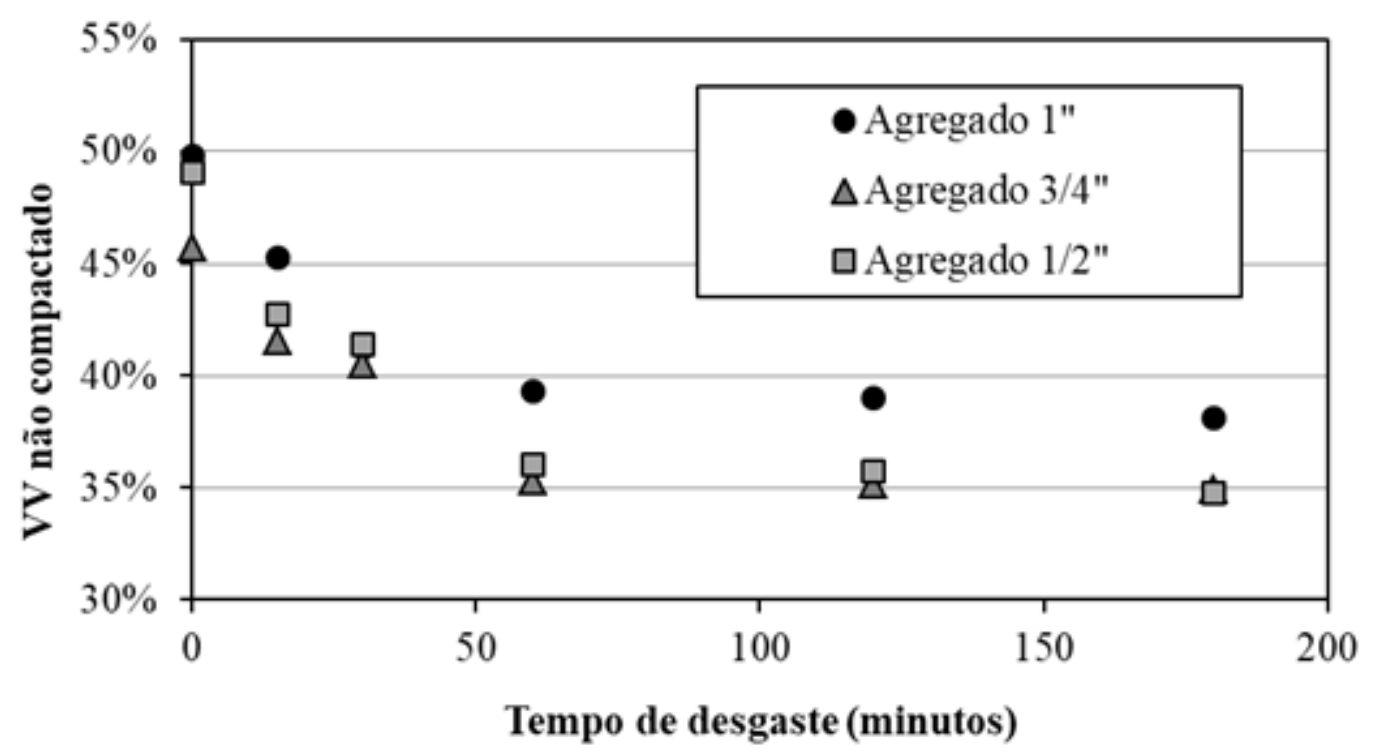

Figura 2: Relação entre Volume de vazios (VV) não compactados e degradação dos agregados por meio do uso do MD.

Os resultados indicam que, para o agregado de 1", houve uma queda acentuada de VV não compactado durante os primeiros 60 minutos de desgaste. Essa perda foi de aproximadamente $11 \%$, enquanto que entre 60 e 180 minutos de polimento, essa queda foi de apenas 3\%. Essas diferenças corroboraram os relatos de MASAD et al. [2], que afirmam que, para as amostras utilizadas e processadas com a mesma carga abrasiva utilizada na metodologia deste trabalho, o agregado tende a atingir condições de degradação terminal a partir de 180 minutos de degradação no MD. Como se pode observar também, existe um comportamento muito similar entre a amostra do agregado de $1 "$ e as amostras dos agregados de 3/4" e de 1/2". Existe uma tendência de queda acentuada no percentual de VV não compactado nos primeiros 60 minutos de degradação e menor perda desse parâmetro entre 60 e 180 minutos.

É importante lembrar que o VV não compactado é um parâmetro cuja obtenção é influenciada por diversas varáveis, desde aquelas que são de interesse deste trabalho (textura superficial, angularidade e forma) a outras que podem mascarar resultados e induzir a outras interpretações (variação na distribuição granulométrica da amostra, por exemplo). Durante o processo de degradação das amostras deste estudo, verificou-se que a quantidade de partículas em cada fração sofreu variações significativas, isto é, as curvas granulométricas das amostras variaram conforme o agregado era degradado. Observou que a curva granulométrica da amostra assumiu um comportamento mais bem distribuído, fazendo com que os vazios fossem ocupados pelas partículas de menor tamanho e diminuindo o VV não compactado da amostra.

A variação de massa ao longo do processo de degradação (Figura 3) pode impactar as propriedades de forma dos agregados. Uma variação acentuada da massa do agregado pode significar que parte do agregado que era retido em uma peneira acaba passando e migrando para peneiras subsequentes. Esse processo pode ocorrer de duas maneiras: a primeira a partir do processo de polimento dos agregados, no qual a partícula é polida a ponto de reduzir tanto o seu tamanho que esta passa a ser retida apenas na peneira imediatamente subsequente; a segunda é que durante o processo de degradação a partícula sofre quebra, devido ao acúmulo de dano sofrido durante o processo e passa a possuir dimensões menores, podendo ser retida em outras peneiras. 


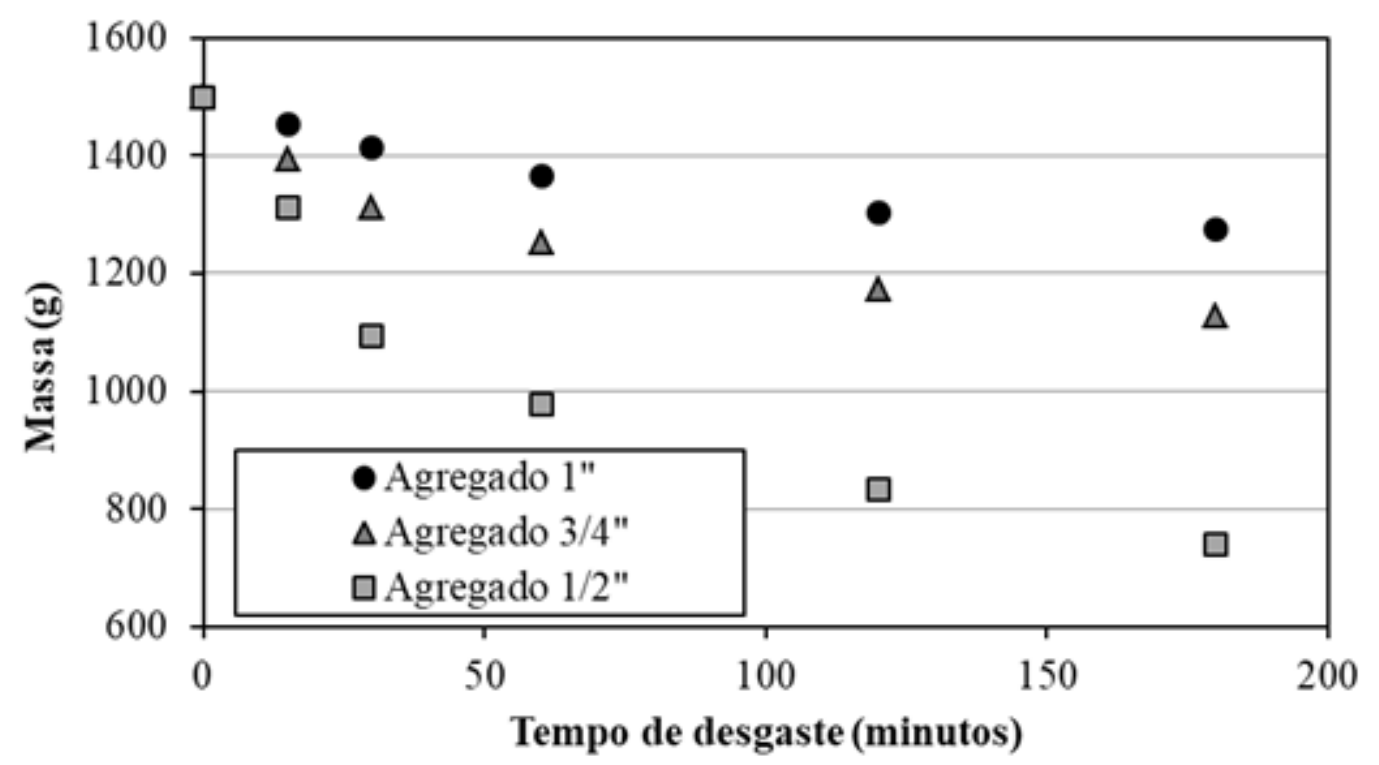

Figura 3: Total de perda de massa dos agregados no MD.

Uma análise geral do que aconteceu com a massa das amostras durante todo o processo de quebra permite concluir que, para os materiais analisados no $\mathrm{MD}$, frações menores de agregados (1/2") tendem a perder massa de maneira mais rápida que os agregados compostos de partículas maiores (1" e 3/4").

O ambiente no qual as partículas são degradadas pode exercer grande influência sobre o processo de degradação. Devido a isso, foi proposta uma etapa do estudo na qual os agregados foram degradados em ambiente molhado, com o objetivo de se verificar a influência da presença de água na perda de propriedades desses materiais. São apresentados resultados de testes realizados nas três frações de agregados (1", 3/4" e $1 / 2$ ") em ambiente úmido, por meio de análises dos mesmos aspectos aos quais foram analisadas as amostras de agregados secos. A Figura 4 mostra a comparação dos resultados de Volume de vazios não compactado e perda de massa entre amostras desgastadas a seco e imersas em água.

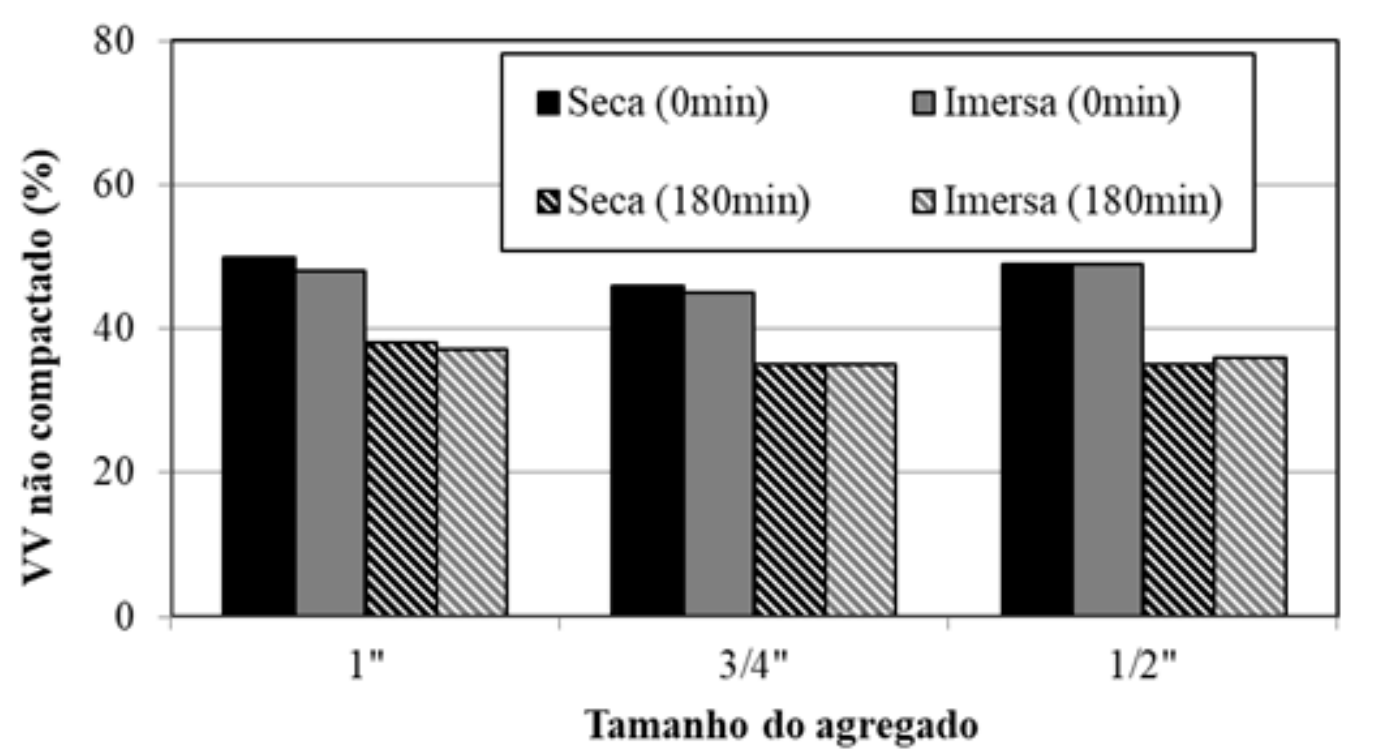

Figura 4: Relação entre o Volume de vazios (Vv) não compactado e o TMN dos agregados para diferentes ambientes (seco e imerso) e tempos de desgaste no MD.

\subsubsection{Variação nas propriedades de forma}

A angularidade está diretamente relacionada com a quantidade de faces fraturadas presentes nas partículas 
dos agregados. Isso significa que, quanto maior for o número de planos de quebra da superfície da partícula do agregado, maior será o valor de angularidade do material. Dessa forma, cada amostra de agregado foi analisada com o uso do AIMS2, para que os valores de angularidade pudessem ser medidos. A Tabela 4 apresenta os resultados obtidos para as frações antes e após 180 minutos de desgaste no MD.

Tabela 4: Perda de angularidade das frações dos agregados desgastados no MD.

\begin{tabular}{|c|c|c|c|c|}
\hline \multirow[t]{2}{*}{ TMN } & \multirow[t]{2}{*}{ FRAÇÃO } & \multicolumn{2}{|c|}{ TEMPO DE DEGRADAÇÃO (MIN.) } & \multirow[t]{2}{*}{ PERDA DE ANGULARIDADE (\%) } \\
\hline & & 0 & 180 & \\
\hline \multirow[t]{6}{*}{$1 "$} & \multirow[t]{2}{*}{$5 / 8 "$} & 2.880 & 2.170 & \multirow[t]{2}{*}{25} \\
\hline & & Subarredondado & Subarredondado & \\
\hline & \multirow[t]{2}{*}{$1 / 2^{\prime \prime}$} & 2.776 & 2.135 & \multirow[t]{2}{*}{23} \\
\hline & & Subarredondado & Subarredondado & \\
\hline & \multirow[t]{2}{*}{ 3/8" } & 3.016 & 2.270 & \multirow[t]{2}{*}{25} \\
\hline & & Subarredondado & Subarredondado & \\
\hline \multirow[t]{6}{*}{$3 / 4 "$} & \multirow[t]{2}{*}{$3 / 8^{\prime \prime}$} & 2.793 & 2.058 & \multirow[t]{2}{*}{26} \\
\hline & & Subarredondado & Arredondado & \\
\hline & \multirow[t]{2}{*}{$1 / 4^{\prime \prime}$} & 3.041 & 2.085 & \multirow[t]{2}{*}{31} \\
\hline & & Subarredondado & Arredondado & \\
\hline & \multirow[t]{2}{*}{ \#4 } & 2.979 & 1.840 & \multirow[t]{2}{*}{38} \\
\hline & & Subarredondado & Arredondado & \\
\hline \multirow[t]{4}{*}{$1 / 2^{\prime \prime}$} & \multirow[t]{2}{*}{$1 / 4^{\prime \prime}$} & 3.028 & 1.951 & \multirow[t]{2}{*}{36} \\
\hline & & Subarredondado & Arredondado & \\
\hline & \multirow[t]{2}{*}{$\# 4$} & 3.169 & 1.911 & \multirow[t]{2}{*}{40} \\
\hline & & Subarredondado & Arredondado & \\
\hline
\end{tabular}

No geral, a angularidade média das partículas de cada amostra diminuiu. No entanto, o que se observa é uma relativa resistência à mudança de classificação das partículas analisadas, apesar de serem observadas reduções de angularidade da ordem de 40\%, (fração de \#4 da amostra de TMN igual a 1/2") que teve sua classificação alterada após 180 minutos de polimento. A observação de frações de mesmo tamanho permitiu concluir que os agregados com maiores percentuais de partículas menores tendem a apresentar maior redução do valor médio de angularidade. A textura superficial também foi medida, e os resultados obtidos são apresentados na Tabela 5 .

Tabela 5: Perda de textura superficial das frações dos agregados desgastados no MD.

\begin{tabular}{|c|c|c|c|c|}
\hline \multirow[t]{2}{*}{ TMN } & \multirow[t]{2}{*}{ FRAÇÃO } & \multicolumn{2}{|c|}{ TEMPO DE DEGRADAÇÃO (MIN.) } & \multirow[t]{2}{*}{ PERDA DE TEXTURA (\%) } \\
\hline & & $\mathbf{0}$ & 180 & \\
\hline \multirow[t]{6}{*}{ 1" } & \multirow[t]{2}{*}{$5 / 8^{\prime \prime}$} & 298 & 228 & \multirow[t]{2}{*}{23} \\
\hline & & Baixa rugosidade & Liso & \\
\hline & \multirow[t]{2}{*}{$1 / 2^{\prime \prime}$} & 304 & 193 & \multirow[t]{2}{*}{37} \\
\hline & & Baixa rugosidade & Liso & \\
\hline & \multirow[t]{2}{*}{$3 / 8^{\prime \prime}$} & 280 & 193 & \multirow[t]{2}{*}{31} \\
\hline & & Baixa rugosidade & Liso & \\
\hline \multirow[t]{5}{*}{$3 / 4 "$} & \multirow[t]{2}{*}{ 3/8" } & 199 & 151 & \multirow[t]{2}{*}{24} \\
\hline & & Liso & Liso & \\
\hline & \multirow[t]{2}{*}{$1 / 4^{\prime \prime}$} & 201 & 124 & \multirow[t]{2}{*}{38} \\
\hline & & Liso & Polido & \\
\hline & $\# 4$ & 125 & 136 & -9 \\
\hline
\end{tabular}




\begin{tabular}{l|l|l|l|l}
\hline & & Polido & Polido & \\
\hline $\mathbf{1} / \mathbf{2}^{\mathbf{1}}$ & \multirow{2}{*}{$\mathbf{4} "$} & 203 & 150 & 26 \\
\cline { 3 - 4 } & Liso & Polido & \\
\cline { 2 - 4 } & \multirow{2}{*}{$\mathbf{4}$} & 197 & 151 & 23 \\
\cline { 3 - 5 } & & Liso & Polido & \\
\hline
\end{tabular}

É possível perceber que, em relação ao comportamento dos valores médios de textura superficial das frações dos agregados analisados, houve perda dessa propriedade para todas as frações, exceto para um caso (fração de \#4 do agregado de tamanho de 3/4"), porém a variação nesta propriedade foi baixa. O valor negativo pode ser explicado pela variação intrínseca no próprio ensaio realizado no AIMS2 para a obtenção dessa propriedade, indicando que não houve necessariamente uma mudança no valor médio obtido antes e depois do uso do MD. Comparando as frações que se repetem dentro das amostras, é possível concluir que, em geral, as amostras com maiores TMN tendem a ter uma maior redução de textura superficial para o tempo de polimento analisado, o que é similar ao que ocorreu em relação à perda de massa das amostras. Como apresentado anteriormente, as amostras com maiores TMN tendem a perder menos massa durante a degradação no MD. Isso pode significar que, durante o desgaste, as partículas maiores tendem a sofrer mais polimento do que quebra, o que pode ser explicado por serem menos suscetíveis à carga abrasiva. A Tabela 6 apresenta os resultados de perda da propriedade de esfericidade.

Tabela 6: Perda de esfericidade das frações dos agregados desgastados no MD.

\begin{tabular}{|c|c|c|c|c|}
\hline \multirow[t]{2}{*}{ TMN } & \multirow[t]{2}{*}{ FRAÇÃO } & \multicolumn{2}{|c|}{ TEMPO DE DEGRADAÇÃO (MIN.) } & \multirow[t]{2}{*}{ PERDA DE ESFERICIDADE (\% } \\
\hline & & $\mathbf{0}$ & 180 & \\
\hline \multirow[t]{6}{*}{ 1" } & \multirow[t]{2}{*}{$5 / 8^{\prime \prime}$} & 0,71 & 0,71 & \multirow[t]{2}{*}{0} \\
\hline & & Esfericidade moderada & Esfericidade moderada & \\
\hline & \multirow[t]{2}{*}{$1 / 2^{\prime \prime}$} & 0,67 & 0,70 & \multirow[t]{2}{*}{-4} \\
\hline & & Baixa esfericidade & Esfericidade moderada & \\
\hline & \multirow[t]{2}{*}{$3 / 8^{\prime \prime}$} & 0,62 & 0,64 & \multirow[t]{2}{*}{-3} \\
\hline & & Baixa esfericidade & Baixa esfericidade & \\
\hline \multirow[t]{6}{*}{$3 / 4^{\prime \prime}$} & \multirow[t]{2}{*}{$3 / 8^{\prime \prime}$} & 0,74 & 0,73 & \multirow[t]{2}{*}{1} \\
\hline & & Esfericidade moderada & Esfericidade moderada & \\
\hline & \multirow[t]{2}{*}{$1 / 4^{\prime \prime}$} & 0,66 & 0,72 & \multirow[t]{2}{*}{-9} \\
\hline & & Baixa esfericidade & Esfericidade moderada & \\
\hline & \multirow[t]{2}{*}{$\# 4$} & 0,66 & 0,73 & \multirow[t]{2}{*}{-11} \\
\hline & & Baixa esfericidade & Esfericidade moderada & \\
\hline \multirow[t]{4}{*}{$1 / 2^{\prime \prime}$} & \multirow[t]{2}{*}{$1 / 4^{\prime \prime}$} & 0,67 & 0,71 & \multirow[t]{2}{*}{-6} \\
\hline & & Baixa esfericidade & Esfericidade moderada & \\
\hline & \multirow[t]{2}{*}{$\# 4$} & 0,67 & 0,75 & \multirow[t]{2}{*}{-12} \\
\hline & & Baixa esfericidade & Esfericidade moderada & \\
\hline
\end{tabular}

Os resultados apresentados pelas esfericidades médias de cada uma das frações apenas corroboram o que o estudo da distribuição percentual das partículas entre as classes apresentou. O desgaste provocado pelo MD nas amostras ao longo do tempo de polimento aplicado foi insuficiente para provocar alterações significativas na esfericidade das partículas. Além disso, houve um aumento na esfericidade para algumas amostras, conforme indicado pelos valores negativos de perda de propriedade, que indicam um aumento no valor desse parâmetro. A Figura 5 apresenta os resultados de variação nas propriedades de forma com e sem a imersão em água no equipamento MD. 


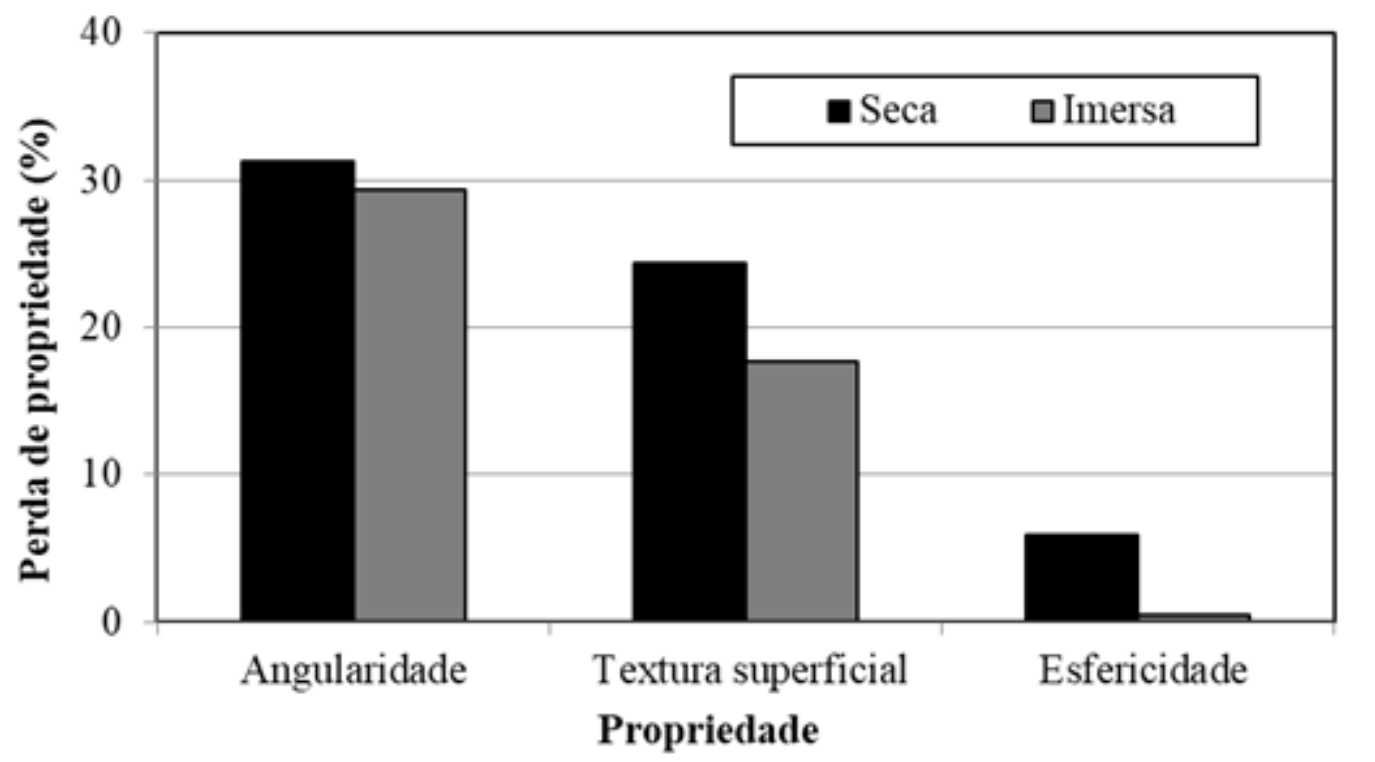

Figura 5: Perda de propriedades de forma no Micro-Deval, para agregados em condição seca e condição imersa.

\subsection{Análise das amostras desgastadas no Micro-Deval (MD)}

Apesar de haver semelhanças entre o uso dos equipamentos LA e MD, sabe-se que o primeiro provoca um processo de desgaste muito severo aos agregados, levando à quebra dos materiais e não necessariamente à abrasão e ao polimento. Acredita-se que o ensaio realizado no MD é capaz de submeter as amostras a processos que remetem à mudanças nas propriedades de forma dos materiais e não apenas à quebra das partículas. Sendo assim, foram comparados os resultados obtidos em ambos os equipamentos, para as propriedades analisadas neste trabalho.

\subsubsection{Perda de massa e Volume de vazios (VV) não compactados}

O primeiro dado analisado foi o percentual de perda de massa, que é um dos objetivos principais da norma de ensaio de abrasão. Desta forma, as perdas de massa das amostras de agregados em estudo, após o uso do equipamento de LA foram de 29, 35 e 37\% para os TMNs de 1", 3/4" e 1/2", respectivamente, indicando que os três materiais estão dentro das normas comumente adotados para agregados de misturas asfálticas, que exigem, no geral, valores máximos de 40 ou $45 \%$.

Observou-se uma significativa perda de massa principalmente para os agregados que possuem partículas menores. Apesar da grande redução, os agregados ainda podem ser utilizados em serviços de pavimentação, segundo o manual de pavimentação do DNIT (DNIT, 2010), que preconiza que agregados com até 40\% de redução de massa sejam utilizados para pavimentação sem a necessidade de comprovar a eficiência do agregado na mistura. Comparando os resultados de perda de massa obtidos pelo uso do equipamento LA com aqueles obtidos pelo uso do MD, foi possível observar que para a amostra de 1", mesmo ao final do período de processo de desgaste do agregado no MD, a perda de massa deste agregado foi maior no LA, de modo que a amostra de agregado submersa foi a que mais se aproximou, apresentando uma redução de massa de $22 \%$. Para o agregado de 3/4" a redução percentual de massa foi superior, tornando distante qualquer comparação entre as reduções resultantes do desgaste no MD. Porém, para a amostra de TMN igual a 1/2", observou-se que a amostra submersa processada no MD se aproximou do valor obtido ao final do período de desgaste, apresentando uma redução de $36 \%$ da massa, já a amostra ensaiada sem a presença de água apresentou esse percentual de redução de massa no intervalo entre $60(35 \%)$ e 120 minutos (44\%) de desgaste. A Figura 6 apresenta os resultados de Volume de vazios (Vv) não compactados após o uso do LA. 


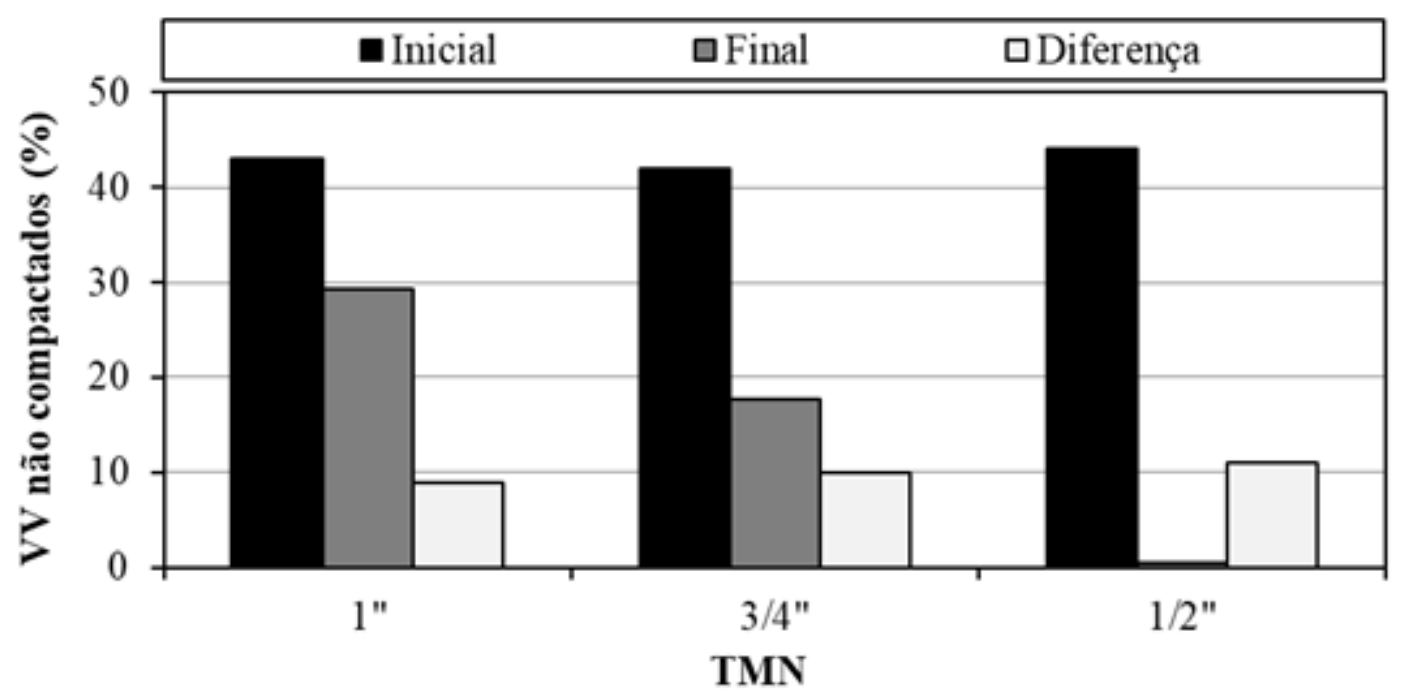

Figura 6: Volume de Vazios (VV) não compactados (abrasão Los Angeles).

A partir dos resultados de VV não compactados, observou-se que os agregados cujas partículas tinham menor tamanho apresentaram redução maior do percentual de VV, tanto para as partículas processadas no LA, quanto para as partículas processadas no MD. Porém essa redução do VV é observada nos primeiros 15 minutos de desgaste da amostra, seja ela submersa ou não, no MD.

\subsubsection{Variações nas propriedades de forma}

As propriedades de forma foram analisadas antes e depois o uso do equipamento LA. A Tabela 7 apresenta os resultados de perda de angularidade obtidos para cada uma das frações.

Tabela 7: Perda de angularidade das frações dos agregados degradados (abrasão Los Angeles).

\begin{tabular}{|c|c|c|c|c|}
\hline TMN & FRAÇÃO & ANTES & APós & PERDA DE ANGULARIDADE (\%) \\
\hline \multirow[t]{6}{*}{$1^{\prime \prime}$} & \multirow[t]{2}{*}{$5 / 8^{\prime \prime}$} & 2.880 & 2.221 & \multirow[t]{2}{*}{23} \\
\hline & & Subarredondado & Subarredondado & \\
\hline & \multirow[t]{2}{*}{$1 / 2^{\prime \prime}$} & 2.776 & 2.381 & \multirow[t]{2}{*}{14} \\
\hline & & Subarredondado & Subarredondado & \\
\hline & \multirow[t]{2}{*}{$3 / 8^{\prime \prime}$} & 3.016 & 2.311 & \multirow[t]{2}{*}{23} \\
\hline & & Subarredondado & Subarredondado & \\
\hline \multirow[t]{6}{*}{$3 / 4^{\prime \prime}$} & \multirow[t]{2}{*}{$3 / 8^{\prime \prime}$} & 2.793 & 2.285 & \multirow[t]{2}{*}{18} \\
\hline & & Subarredondado & Subarredondado & \\
\hline & \multirow[t]{2}{*}{$1 / 4^{\prime \prime}$} & 3.041 & 2.382 & \multirow[t]{2}{*}{22} \\
\hline & & Subarredondado & Subarredondado & \\
\hline & \multirow[t]{2}{*}{$\# 4$} & 2.979 & 2.531 & \multirow[t]{2}{*}{15} \\
\hline & & Subarredondado & Subarredondado & \\
\hline \multirow[t]{4}{*}{$1 / 2^{\prime \prime}$} & \multirow[t]{2}{*}{$1 / 4^{\prime \prime}$} & 3.028 & 2.676 & \multirow[t]{2}{*}{12} \\
\hline & & Subarredondado & Subarredondado & \\
\hline & \multirow[t]{2}{*}{$\# 4$} & 3.169 & 2.547 & \multirow[t]{2}{*}{20} \\
\hline & & Subarredondado & Subarredondado & \\
\hline
\end{tabular}

Conforme observado, a angularidade média das frações diminui. Essa redução foi bem mais perceptível no agregado de 1 " seguido pelo agregado de 3/4" e por último o agregado de $1 / 2$ ", ao contrário do que ocorre com a angularidade das frações de cada amostra no MD, em que as amostras de agregados de menor tamanho sofrem maior perda de angularidade. A Tabela 8 mostra os resultados de perda de textura superficial. 
Tabela 8: Perda de textura superficial das frações dos agregados degradados (abrasão Los Angeles).

\begin{tabular}{|c|c|c|c|c|}
\hline TMN & FRAÇÃO & ANTES & APÓS & PERDA DE TEXTURA $(\%)$ \\
\hline \multirow[t]{6}{*}{ 1" } & \multirow[t]{2}{*}{$5 / 8^{\prime \prime}$} & 298 & 250 & \multirow[t]{2}{*}{16} \\
\hline & & Baixa rugosidade & Liso & \\
\hline & \multirow[t]{2}{*}{$1 / 2^{\prime \prime}$} & 304 & 302 & \multirow[t]{2}{*}{1} \\
\hline & & Baixa rugosidade & Baixa rugosidade & \\
\hline & \multirow[t]{2}{*}{ 3/8" } & 280 & 203 & \multirow[t]{2}{*}{28} \\
\hline & & Baixa rugosidade & Liso & \\
\hline \multirow[t]{6}{*}{ 3/4" } & \multirow[t]{2}{*}{$3 / 8^{\prime \prime}$} & 199 & 200 & \multirow[t]{2}{*}{-1} \\
\hline & & Liso & Liso & \\
\hline & \multirow[t]{2}{*}{$1 / 4^{\prime \prime}$} & 201 & 197 & \multirow[t]{2}{*}{2} \\
\hline & & Liso & Liso & \\
\hline & \multirow[t]{2}{*}{$\# 4$} & 125 & 136 & \multirow[t]{2}{*}{-9} \\
\hline & & Liso & Polido & \\
\hline \multirow[t]{4}{*}{$1 / 2 "$} & \multirow[t]{2}{*}{$1 / 4^{\prime \prime}$} & 203 & 191 & \multirow[t]{2}{*}{6} \\
\hline & & Liso & Liso & \\
\hline & \multirow[t]{2}{*}{ \#4 } & 197 & 143 & \multirow[t]{2}{*}{27} \\
\hline & & Liso & Polido & \\
\hline
\end{tabular}

Foi possível verificar uma pequena variação dos valores de textura superficial média ao se comparar os agregados antes e após o uso do equipamento LA, para a maioria das frações analisadas. Os resultados da perda de esfericidade encontram-se na Tabela 9. Observa-se que, ao serem submetidas aos processos de degradação no equipamento LA, o valor médio de esfericidade aumentou para todas as frações, inclusive fazendo com que a classificação de alguma delas mudasse.

Tabela 9: Perda de esfericidade das frações dos agregados degradados (abrasão Los Angeles).

\begin{tabular}{|c|c|c|c|c|}
\hline TMN & FRAÇÃO & ANTES & APÓS & PERDA DE ESFERICIDADE (\%) \\
\hline \multirow[t]{6}{*}{ 1" } & \multirow[t]{2}{*}{$5 / 8 "$} & 0,71 & 0,77 & \multirow[t]{2}{*}{-8} \\
\hline & & Esfericidade moderada & Esfericidade moderada & \\
\hline & \multirow[t]{2}{*}{$1 / 2^{\prime \prime}$} & 0,67 & 0,76 & \multirow[t]{2}{*}{-13} \\
\hline & & Baixa esfericidade & Esfericidade moderada & \\
\hline & \multirow[t]{2}{*}{$3 / 8^{\prime \prime}$} & 0,62 & 0,75 & \multirow[t]{2}{*}{-21} \\
\hline & & Baixa esfericidade & Esfericidade moderada & \\
\hline \multirow[t]{6}{*}{ 3/4" } & \multirow[t]{2}{*}{ 3/8" } & 0,74 & 0,77 & \multirow[t]{2}{*}{-4} \\
\hline & & Esfericidade moderada & Esfericidade moderada & \\
\hline & \multirow[t]{2}{*}{$1 / 4^{\prime \prime}$} & 0,66 & 0,75 & \multirow[t]{2}{*}{-14} \\
\hline & & Baixa esfericidade & Esfericidade moderada & \\
\hline & \multirow[t]{2}{*}{$\# 4$} & 0,66 & 0,73 & \multirow[t]{2}{*}{-11} \\
\hline & & Baixa esfericidade & Esfericidade moderada & \\
\hline \multirow[t]{4}{*}{ 1/2" } & \multirow[t]{2}{*}{$1 / 4^{\prime \prime}$} & 0,67 & 0,72 & \multirow[t]{2}{*}{-7} \\
\hline & & Baixa esfericidade & Esfericidade moderada & \\
\hline & \multirow[t]{2}{*}{$\# 4$} & 0,67 & 0,75 & \multirow[t]{2}{*}{-12} \\
\hline & & Baixa esfericidade & Esfericidade moderada & \\
\hline
\end{tabular}

3.4 Comparação entre abrasão Los Angeles e Micro-Deval

A Figura 7 mostra comparações entre a perda das propriedades de forma analisadas após a degradação em 
cada ensaio realizado nesta pesquisa (Micro-Deval e abrasão Los Angeles). É possível observar que o teste realizado no MD modifica mais as propriedades de angularidade e textura superficial, enquanto o LA altera mais a esfericidade das partículas. Além disso, a perda de esfericidade foi negativa para quase todas as frações de agregados, tanto para o MD como para o LA, ou seja, os valores para essa propriedade aumentaram após os processos de degradação.

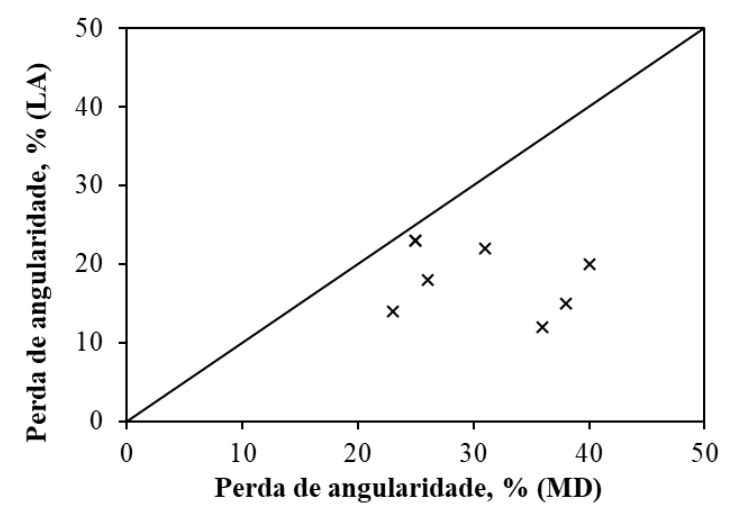

(a)

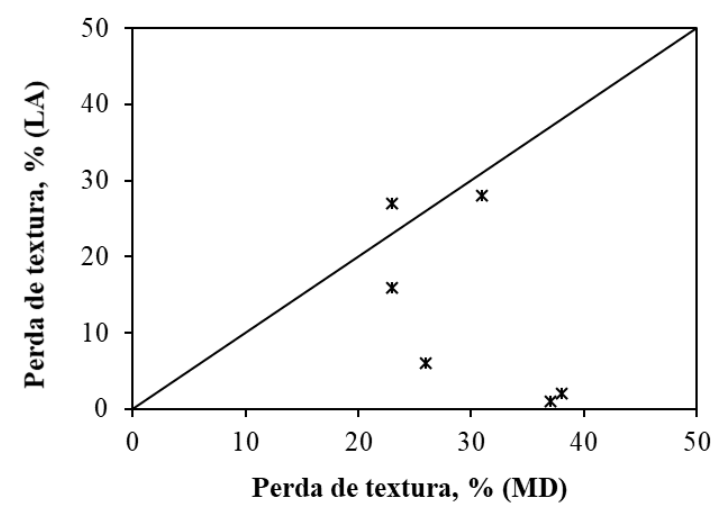

(b)

Perda de esfericidade, \% (MD)

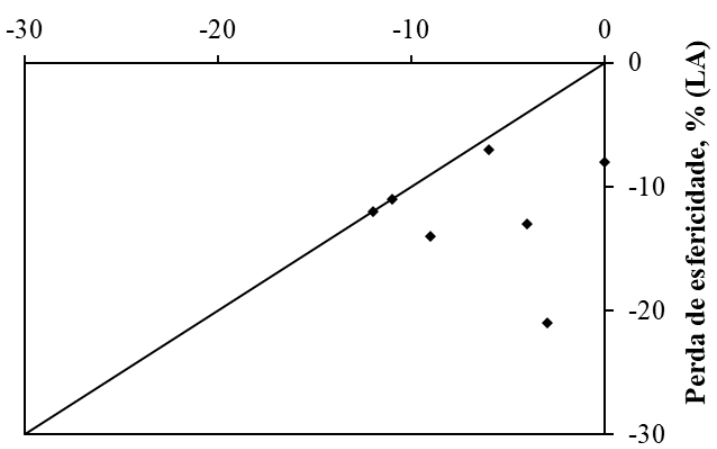

(c)

Figura 7: Comparação entre a perda de propriedades por meio do Micro-Deval e do Los Angeles: (a) angularidade, (b) textura superficial e (c) esfericidade.

\section{CONCLUSÕES}

Este trabalho teve como objetivo principal avaliar o impacto das variações nas propriedades de forma de agregados, analisados após diferentes métodos de desgaste. Com base nos resultados, as seguintes conclusões foram tiradas:

- No processo de degradação das partículas, a composição granulométrica da amostra exerce grande influência na amostra. Amostras com partículas menores apresentam redução de massa e redução de volume de vazios maiores, independente do ambiente (seco ou úmido) a que estiveram submetidas;

- A angularidade das partículas ensaiadas, tanto pelo equipamento Micro-Deval quanto pelo equipamento de abrasão Los Angeles, diminuiu após o processo de desgaste. A angularidade sofre influência da distribuição granulométrica, de modo que amostras com tamanhos de partículas menores tendem a apresentar maiores percentuais de partículas classificadas como arredondadas. Esta propriedade sofre, também, influência do meio em que são degradadas, a amostra de agregado de 1" degradado imerso em água apresentou valores percentuais mais altos de partículas arredondadas. Entretanto, para as amostras de 3/4" e de 1/2" os percentuais mais altos de partículas arredondadas foram encontrados nas partículas degradadas sem a presença da água;

- A textura superficial, quando analisada nas amostras que passam pelo desgaste no LA, não apresentou alterações significativas, uma vez que durante este processo o modo de degradação é, predomi- 
nantemente, o impacto, favorecendo a quebra; a textura, quando analisada nas amostras que passaram pelo MD apresentou comportamentos que variam conforme o tempo de degradação, a distribuição granulométrica do material e o meio no qual as partículas são degradadas. Com o tempo é possível perceber que existe um valor de textura superficial terminal, que não se modificada após algum tempo (variável, a depender da amostra analisada); com isso, o percentual de partículas polidas e lisas tende a aumentar até que durante o processo de degradação o acúmulo de dano nas partículas promove um favorecimento do processo de quebra das faces das partículas maior que o processo de polimento em si. Agregados com distribuições granulométricas menores tendem a possuir maiores percentuais de partículas lisas e polidas ao longo do processo de degradação no MD. Amostras degradadas imersas em água tendem a apresentar maior percentual de partículas com alta rugosidade, rugosidade moderada e baixa rugosidade;

- Os valores de esfericidade nas amostras desgastadas pelo MD não apresentaram variações significativas, independentemente do tempo de degradação, do tamanho das partículas dentro da amostra ou do meio (seco ou úmido) em que foram testadas; a esfericidade para as amostras degradadas pelo LA apresentou perceptíveis variações, apresentando percentual de partículas esféricas maior após o processo de degradação;

- Em termos de análise de resistência à modificação de parâmetros de forma, textura superficial e angularidade, recomenda-se o uso do MD para avaliar a resistência à variação de textura e à variação de angularidade. Para a resistência à variação de forma, recomenda-se o uso do LA Angeles, uma vez que esse equipamento é capaz de alterar significativamente essa propriedade, ao contrário do MD.

\section{AGRADECIMENTOS}

Os autores gostariam de agradecer o apoio da CAPES e do CNPq pelo financiamento da pesquisa e pelo fornecimento de bolsas.

\section{BIBLIOGRAFIA}

[1] MAHMOUD, E. M. "Development of experimental method for the evaluation of aggregate resistance to polish, abrasion, and breakage”, Dissertação de M.Sc., Texas A\&M University, College Station, T.X., EUA, 2005.

[2] MASAD, E., LUCE, A., MAHMOUD, E., "Implementation of aims in measuring aggregate resistance to polishing, abrasion and breakage", In: Report No. FHWA-ICT-14-014, Illinois Department of Transportation (IDOT), Urbana, I.L., EUA, 2006.

[3] BESSA, I.S., CASTELO BRANCO, V.T.F., SOARES, J.B. "Avaliação da resistência ao polimento e à degradação de agregados utilizando a abrasão Los Angeles e o processamento digital de imagens”, In: Anais do $7^{\circ}$ Congresso Brasileiro de Rodovias e Concessões, Foz do Iguaçu, PR, Brasil, 2011.

[4] ARAUJO, V.M.C. “Avaliação da textura de misturas asfálticas a partir do uso do processamento digital de imagens”, Trabalho de Conclusão de Curso, Universidade Federal do Ceará, Fortaleza, CE, Brasil, 2014.

[5] MASAD, E., REZAEI, A., CHOWDHURY, A., HARRIS, P. "Predicting asphalt mixture skid resistance based on aggregate characteristics", In: Report No. 0-5627-1, Texas Department of Transportation (TxDOT), College Station, T.X., EUA, 2009.

[6] SHBEEB, N.I., KHASAWNEH, M.A., AL-OMARI, A.A. "The development of a predictive tool to reduce experimentation time for the polishing and frictional evaluation of asphalt pavement surfaces", Construction and Building Materials, v. 186, pp. 740-750, 2018.

[7] HOU, S., SHI, X., DENG, Y., GU, F. "Evaluation of rutting and friction resistance of hot mix asphalt concrete using an innovative vertically loaded wheel tester", Construction and Building Materials, v. 176, pp. 710-719, 2018.

[8] GEORGIOU, P., LOIZOS, A. "A laboratory compaction approach to characterize asphalt pavement surface friction performance", Wear, v. 311, pp. 114-122, 2014.

[9] MASAD, E., LUCE, A., MAHMOUD, E., CHOWDHURY, A. "Relationship of aggregate texture to asphalt pavement skid resistance using image analysis of aggregate shape”, In: Final Report for Highway IDEA Project 114, College Station, T.X., EUA, 2007. 
[10] KANDHAL, P.S., PARKER JR, F. “Aggregate tests related to asphalt concrete performance in pavements”, In: Report 405, National Cooperative Highway Research Program, NCHRP, Washington, D.C., 1998.

[11] WANG, H., BU, Y., WANG, Y., et al., "The effect of morphological characteristic of coarse aggregates measured with fractal dimension on asphalt mixture's high-temperature performance", Advances in Materials Science and Engineering, v. 2016, pp. 1-10, 2016.

[12] GREER, M., HEITZMAN, M. "Evaluation of the AIMS2 and micro-deval to characterize aggregate friction properties”, In: NCAT Report 17-02, National Center for Asphalt Technology, Auburn, A.L., 2017.

[13] AL-ROUSAN, T., MASAD, E., MEYERS, L., et al., "New methodology for shape classification of aggregates", Transportation Research Record: Journal of the Transportation Research Board, n. 1913, pp. 11$23,2005$.

[14] CROUCH, L., DUNN, T.R. Identification of aggregates for tennessee bituminous surface courses, In: Project Number TNSPR-RES1149, Final Report, Tennessee Department of Transportation (TDOT), Cookeville, T.N., EUA, 2005.

[15] CAVALCANTI, H.P.P. “Avaliação da degradação de propriedades de forma de agregados utilizando o processamento digital de imagens", Trabalho de Conclusão de Curso, Universidade Federal do Ceará, Fortaleza, C.E., Brasil, 2016.

[16] IBIAPINA, D.S. "Proposição de um sistema de classificação das propriedades de forma de agregados caracterizados com o uso do processamento digital de imagens para a seleção de materiais brasileiros", Tese de D.Sc., Universidade Federal do Ceará, Fortaleza, C.E., Brasil, 2018.

\section{ORCID}

Henrique Cavalcanti

Iuri Bessa

Daniele Ibiapina

Verônica Castelo Branco https://orcid.org/0000-0002-8267-4505

https://orcid.org/0000-0002-4711-0552

https://orcid.org/0000-0002-3409-8224

https://orcid.org/0000-0002-1459-1740 